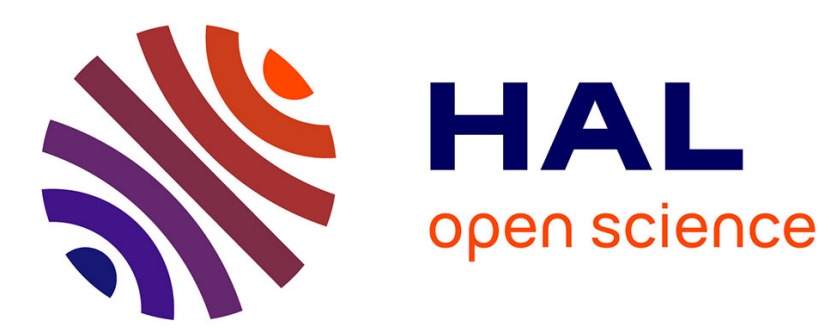

\title{
Length Measurement of Single-Walled Carbon Nanotubes from Translational Diffusion and Intensity Fluctuations
}

\author{
Antony Lee, Laurent Cognet
}

\section{To cite this version:}

Antony Lee, Laurent Cognet. Length Measurement of Single-Walled Carbon Nanotubes from Translational Diffusion and Intensity Fluctuations. Journal of Applied Physics, 2020, 128 (22), pp.224301. 10.1063/5.0031194. hal-02951667

\section{HAL Id: hal-02951667 https://hal.science/hal-02951667}

Submitted on 28 Sep 2020

HAL is a multi-disciplinary open access archive for the deposit and dissemination of scientific research documents, whether they are published or not. The documents may come from teaching and research institutions in France or abroad, or from public or private research centers.
L'archive ouverte pluridisciplinaire HAL, est destinée au dépôt et à la diffusion de documents scientifiques de niveau recherche, publiés ou non, émanant des établissements d'enseignement et de recherche français ou étrangers, des laboratoires publics ou privés. 


\title{
Length Measurement of Single-Walled Carbon Nanotubes from Translational Diffusion and Intensity Fluctuations
}

\author{
Antony Lee ${ }^{1,2}$ and Laurent Cognet ${ }^{1,2, a)}$ \\ 1) Université de Bordeaux, Laboratoire Photonique Numérique et Nanosciences, UMR 5298, 33400 Talence, \\ France \\ 2) Institut d'Optique and CNRS, LP2N UMR 5298, 33400 Talence, France
}

(Dated: 28 September 2020)

\begin{abstract}
A new approach is presented to measure the length distribution of dispersed single-walled carbon nanotubes (SWCNT). In this method, the diffusive trajectories of individual SWCNTs in solution are reconstructed from high-frame rate video stacks. These trajectories allow the estimation of two key statistics for the SWCNTs: their translational diffusion coefficient, and the autocorrelation time of their fluorescence intensity. We show that the autocorrelation time is a measure of the rotational diffusion coefficient of the SWCNTs, and that the length of the SWCNTs can be estimated either from the rotational diffusion coefficients alone, or by combining translational and rotational diffusion coefficients. Moreover, this last estimate does not require knowledge of the solution viscosity or of the SWCNT hydrodynamic diameter.
\end{abstract}

Single-walled carbon nanotubes (SWCNTs) are used in a myriad of applications, including for enhancing the properties of materials ${ }^{1}$, electronics ${ }^{2}$, light sources $^{3}$, bioimaging ${ }^{4}$, or biomedicine ${ }^{5}$, to only name a few. The inherent polydispersity of SWCNTs in their length is a major issue, as different lengths are often preferred for specific applications. While several length sorting methods have been proposed to reduce this dispersity, it remains difficult to estimate the full distribution of SWCNTs lengths in a simple, quantitative, and high yield method.

Atomic force microscopy imaging allows for direct observation of SWCNT length ${ }^{6,7}$, but it is a time-consuming method that requires specialized equipment and skills. In the context of video microscopy experiments, a method that allows the measurement of SWCNT lengths with no additional equipment is highly desirable. The "Length Analysis by Nanotube Diffusion" (LAND) method, proposed by Streit and coworkers $^{8}$, is such a method. Briefly, a LAND experiment consists in imaging single (semiconducting) SWCNTs freely diffusing in an aqueous solution on a fluorescence microscopy setup. Standard localization and linking algorithms allow the reconstruction of continuous SWCNT trajectories ${ }^{9,10}$. The diffusion coefficients of SWCNTs is estimated from these trajectories $^{11-13}$, and their lengths are derived from the diffusion coefficients.

Although LAND is in appearance straightforward, the method requires an accurate estimate of the solution viscosity $\eta$, as the diffusion coefficient is inversely proportional to it. Inaccurate knowledge of the solution viscosity can lead to systematic errors. In particular, the original report and applications of the LAND method reported an up to twofold discrepancy between the SWCNT lengths estimated by LAND and the optically measured length, when considering only SWCNTs so long that their length could be directly measured on the video frames ${ }^{8,14,15}$; this discrepancy was explained as arising from the extreme thinness of the sample-holding chamber ${ }^{16}$, and corrected by replacing the

\footnotetext{
${ }^{a)}$ Electronic mail: laurent.cognet@u-bordeaux.fr
}

bulk solution viscosity by an effective viscosity, whose measurement required a separate calibration. Additionally, for short SWCNTs, which diffuse very quickly in water, highviscosity solutions (glucose or sucrose solutions, or waterglycerol mixtures) may be used to slow down diffusion and simplify SWCNT tracking ${ }^{14,15}$; however, the viscosity of such solutions depends strongly on the mixture fractions and on the temperature ${ }^{17,18}$, which are typically not precisely controlled. For example, between $16^{\circ} \mathrm{C}$ and $32^{\circ} \mathrm{C}$, the viscosity of a $2: 1$ $\mathrm{v} / \mathrm{v}$ water glycerol mixture decreases twofold ${ }^{19}$. Therefore, it would be desirable to be able to make LAND-style analyses independent of the sample viscosity — not only independent of the bulk sample viscosity, which could be measured separately, but also, and more importantly, independent of the effective in situ viscosity. The LAND method also depends on an estimate of the nanotube hydrodynamic diameter. Again, this diameter can typically only be approximated, which is a significant additional source of errors.

Here, we show that any LAND experiment performed at a sufficiently high frame rate already contains additional information that allows estimation of SWCNT length independently of the solution viscosity, and nearly independently of the nanotube hydrodynamic diameter. Specifically, when the SWCNTs are localized, a byproduct of the fitting routine is the SWCNT fluorescence intensity. Here, we show that fluctuations of this intensity along the trajectory report on the SWCNT rotational diffusion coefficient, and that SWCNT length can be estimated either from intensity fluctuations alone, or by combining translational diffusion and intensity fluctuations; most interestingly, this last method does not require knowledge of the solution viscosity or of the SWCNT hydrodynamic diameter.

\section{THEORY}

\section{A. Translational and rotational diffusion}

In a viscous medium, the two-dimensional mean-square displacement of a diffusing SWCNT, 


$$
\operatorname{MSD}_{T}(t)=\frac{1}{T-t} \sum_{i=1}^{T-t}\left(\mathbf{r}_{i+t}-\mathbf{r}_{i}\right)^{2},
$$

(where $T$ is the number of frames in the movie stack and $\mathbf{r}$ 2D projection of the position vector of the SWCNT) grows linearly with the time lag $t$ :

$$
\operatorname{MSD}_{T}(t)=4 D_{T} t
$$

where $D_{T}$ is the translational diffusion coefficient. In the presence of localization error with standard deviation $\sigma$ and motion blur over the exposure time $t_{E}$, the empirically measured mean-square displacement is

$$
\operatorname{MSD}_{T}(t)=4 D_{T} t+4 \sigma^{2}-\frac{4}{3} D_{T} t_{E}
$$

It is well established that due to the finite length of the movies stacks, the optimal estimator for $D_{T}$ consists in computing the slope in an unweighted linear fit for $t$ versus $\operatorname{MSD}(t)$ for the first few time lags ${ }^{8,11}$.

The diffusion of SWCNTs shorter than $3 \mu \mathrm{m}$ can be approximated as the one of rigid rods ${ }^{20,21}$, for which the translational diffusion coefficient is given by

$$
D_{T}=\frac{k T}{3 \pi \eta L}\left[\log p+X_{T}(p)\right] .
$$

where $k$ is the Boltzmann constant, $T$ the temperature, $\eta$ the viscosity of the medium, $L$ the length of the SWCNT, and $p=$ $L / d$ its hydrodynamic aspect ratio (the ratio of its length to its hydrodynamic diameter $d) ; X_{T}(p)$ is a finite-length correction term $^{22}$ :

$X_{T}(p) \approx 0.38-\frac{1.16}{\sqrt{p}}+\frac{1.77}{p}-\frac{0.14}{p^{2}}+\frac{0.55 \log p}{p}-\frac{0.10 \log p}{p^{2}}$.

Equation 4 implicitly defines the LAND length estimate, $L_{T}$, as a function of the translational diffusion coefficient $D_{T}$.

The mean-square angular displacement of a diffusing SW$\mathrm{CNT}, \mathrm{MSD}_{R}(t)$, also grows linearly with time at short time lags,

$$
\operatorname{MSD}_{R}(t)=\frac{1}{T-t} \sum_{i=1}^{T-t}\left(\Delta \alpha_{i, i+t}\right)^{2}=4 D_{R} t,
$$

where $\Delta \alpha_{i, i+t}$ is the change in the direction in which the SWCNT points between times $i$ and $i+t$, and $D_{R}$ is the rotational diffusion coefficient. Similarly to $D_{T}, D_{R}$ can be expressed as

$$
D_{R}(t)=\frac{3 k T}{\pi \eta L^{3}}\left[\ln p+X_{R}(p)\right],
$$

where $X_{R}(p)$ is again a finite-length correction term ${ }^{22}$ :

$$
X_{R}(p) \approx-0.49-\frac{1.18}{\sqrt{p}}+\frac{3.17}{p}-\frac{3.29}{p^{2}}+\frac{3.69}{p^{3}}-\frac{1.72}{p^{4}} .
$$

Assuming that an estimate of $D_{R}$ is available, then, similarly to the translational case, equation 7 implicitly defines a length estimate $L_{R}$ based on the rotational diffusion coefficient $D_{R}$.
Taken together, these expressions suggest a third method for obtaining the length of the SWCNT from experimental estimates of its linear and rotational diffusion coefficients. Namely, we have

$$
\left(\frac{\log p+X_{T}(p)}{\log p+X_{R}(p)}\right)^{1 / 2} L=3\left(\frac{D_{T}}{D_{R}}\right)^{1 / 2},
$$

which implicitly defines the length estimate $L_{T / R}$ as a function of $D_{T}, D_{R}$, and $d$ (remember that $p=L / d$ ). Unlike $L_{T}$ and $L_{R}$, the $L_{T / R}$ estimate is independent of the viscosity $\eta$. $L_{T / R}$ also depends much more weakly on the SWCNT hydrodynamic diameter $d$ when compared to the standard LAND $L_{T}$ estimate. Indeed, consider a typical estimate of the hydrodynamic diameter ${ }^{8}, d=(5 \pm 2) \mathrm{nm}$. Assuming a value of $d=5 \mathrm{~nm}$ when the true value is either $d=3 \mathrm{~nm}$ or $d=7 \mathrm{~nm}$ results in a systematic error of $10 \%$ to $20 \%$ on $L_{T}$ and $L_{R}$ (on the same range as the reported accuracy of $\mathrm{LAND}^{8}$ ), but an order of magnitude less (1\% to $3 \%$ ) on $L_{T / R}$.

\section{B. Fluorescence autocorrelation time}

The estimation of the translational diffusion coefficient from reconstructed experimental trajectories is well understood $^{8,11}$. On the other hand, estimating the rotational diffusion coefficient requires, on the face of it, to obtain the orientation of the SWCNT on each frame, which can only be done if the SWCNT is indeed long enough to be visualized as a non-point emitter on a diffraction-limited imaging setup ( $2500 \mathrm{~nm}$ ). In that case, the length of the SWCNT could be directly measured from its image, and the analysis proposed here is moot (but see discussion regarding optical lengths below).

One could consider using linearly polarized excitation and/or performing polarization-sensitive detection in order to obtain information about the SWCNT orientation. Here, we restrict ourselves to the experimentally simpler case of circularly polarized excitation and polarization-insensitive detection. In fact, the rotational diffusion coefficient can in this case also be indirectly inferred from fluctuations in the SWCNT fluorescence intensity. Indeed, the main cause of fluctuations in the fluorescence intensity is the change in the out-of-plane orientation of the SWCNT.

On the one hand, under circularly polarized excitation, the absorption cross-section of the SWCNT - which can be modelled as a short dipole absorber/emitter with the transition dipole parallel to the nanotube axis - is proportional to $\sin ^{2} \theta$, where $\theta$ is the angle of the SWCNT relative to the optical axis (figure 1a). On the other hand, the fraction of the fluorescence radiated in the detection cone, which is also the collected fraction of the total SWCNT fluorescence (under polarization-insensitive detection), is proportional to

$$
f(\theta)=\frac{1}{2}\left(\cos ^{2} \Theta+\cos \Theta\right)\left(\frac{1}{3}-\cos ^{2} \theta\right)+\frac{2}{3},
$$

where $\Theta=\arcsin (N A / n)$ is the aperture angle of the detection cone, which depends on $N A$, the objective numerical aperture, 
(a)

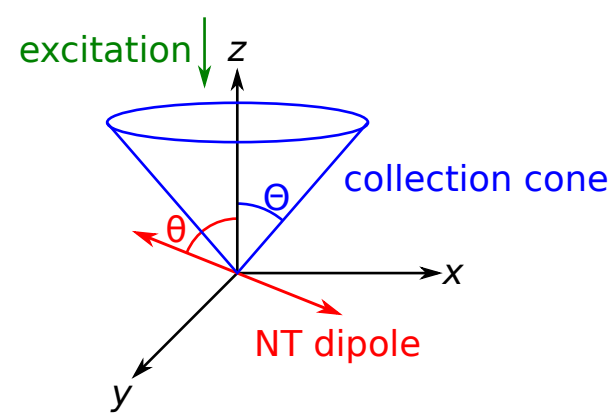

(b)

(c)
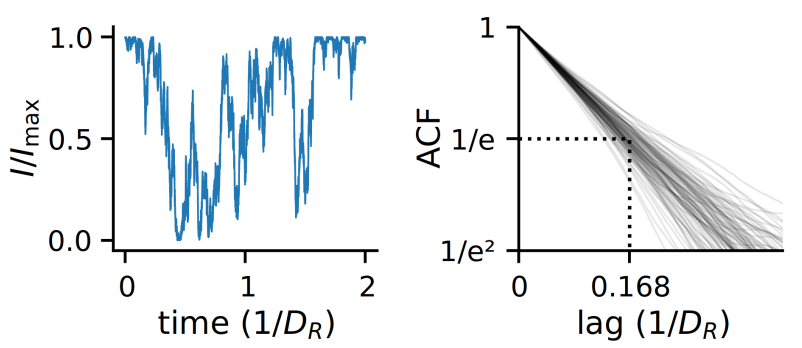

FIG. 1. (a) Experimental geometry. The excitation and fluorescence collection take place along the $z$ axis. (b) Sample simulated fluorescence intensity trace. (c) Fluorescence autocorrelation functions of 100 simulated traces (semi-log $y$ scale). In (b) and (c), times are in units of $1 / D_{R}$, the natural characteristic timescale for rotational diffusion.

and $n$, the index of refraction of the immersion medium ${ }^{14}$. The total observed fluorescence intensity therefore varies as $I(\theta)=f(\theta) \sin ^{2} \theta$.

This expression can be plugged into a rotational random walk simulation to generate theoretical fluorescence time traces, $I(t)$ (figure $1 \mathrm{~b}$ ). By fitting the autocorrelation function of $I(t), \operatorname{ACF}(\Delta t)=\langle I(t) I(t+\Delta t)\rangle /\left\langle I(t)^{2}\right\rangle$ (figure 1c), in the region $\exp (-2)<\operatorname{ACF}(t)<1$, to an exponential model $\operatorname{ACF}(\Delta t)=\exp \left(-\Delta t / \tau_{\mathrm{FA}}\right)$, we find that the fluorescence autocorrelation time $\tau_{\mathrm{FA}}$ satisfies

$$
\tau_{\mathrm{FA}} \approx 0.168 / D_{R}
$$

In other words, the rotational diffusion coefficient can be obtained from the fluorescence autocorrelation time by the simple relation $D_{R}=0.168 / \tau_{\mathrm{FA}}$.

The translational diffusion of SWCNT along the optical axis (in and out of the depth of field) may appear to also contribute to fluctuations in fluorescence intensity. However, the mean time over which a SWCNT diffuses over the depth of field $\mathrm{DOF}(\approx 1 \mu \mathrm{m})$ is $\tau_{\mathrm{DOF}} \approx \mathrm{DOF}^{2} / 2 D_{T}$; therefore, we have

$$
\frac{\tau_{\mathrm{DOF}}}{\tau_{\mathrm{FA}}} \approx 54\left(\frac{\mathrm{DOF}}{L}\right)^{2} \gg 1 .
$$

Hence, the fluctuations in fluorescence intensity due to the translational diffusion of the SWCNT along the optical axis occur over a much longer time scale than rotational diffusion, and can be neglected here.

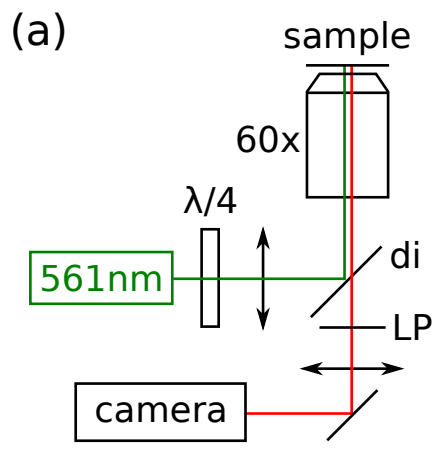

(c)

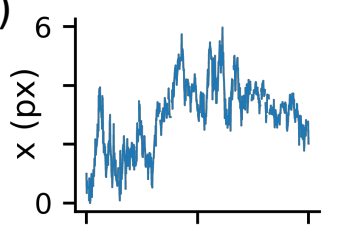

(b)
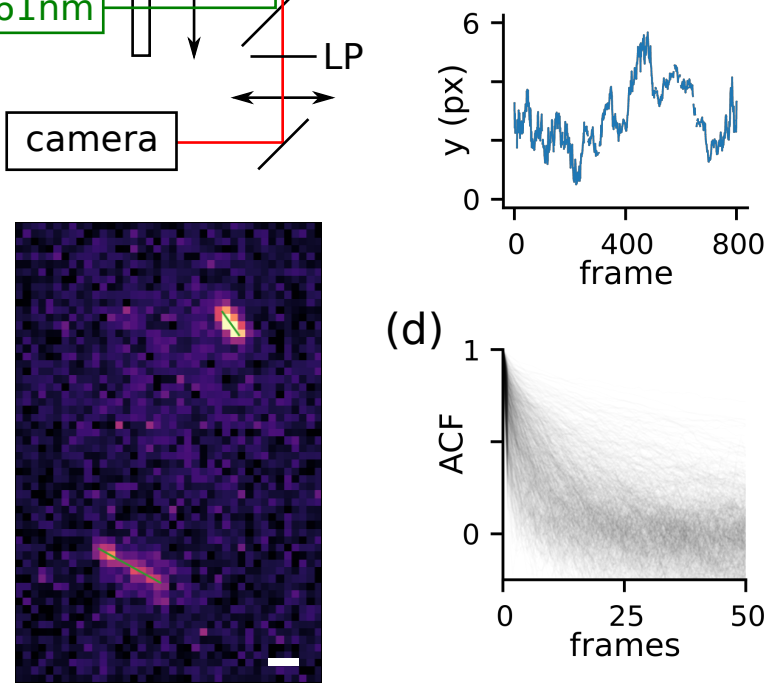

(d)

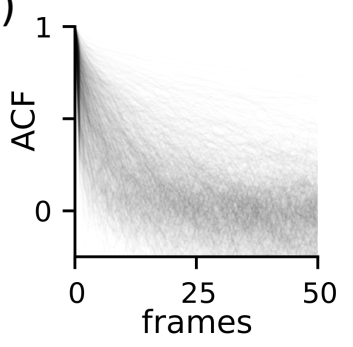

FIG. 2. (a) Microscopy setup for excitation and imaging of $(6,5) \mathrm{SW}$ CNTs. $561 \mathrm{~nm}$ : excitation laser; $\lambda / 4$ : quarter-wave plate; di: $875 \mathrm{~nm}$ long-pass dichroic mirror; LP: $900 \mathrm{~nm}$ long-pass emission filter. (b) Sample frame, including two SWCNTs (fitted to green segments). Scale bar, $1 \mu \mathrm{m}$. (c) Sample SWCNT position ( $x$ vs. $t, y$ vs. $t$ ) traces. (d) Fluorescence autocorrelation functions of all trajectories in the dataset.

\section{ANALYSIS AND DISCUSSION}

In order to validate the $L_{R}$ and $L_{T / R}$ methods, and to compare their performance with the $L_{T}$ (LAND) method, we recorded and analyzed 5 movies, of 8000 frames each, of $(6,5)$ SWCNTs diffusing in a 2:1 v/v glycerol-water mixture (tabulated viscosity ${ }^{19}: \eta=20.6 \mathrm{mPas}$ ). The SWCNTs were excited at the $S_{22}$ transition with a $568 \mathrm{~nm}$ laser. Imaging was performed on a high-speed, near-infrared fluorescence microscopy setup (figure 2a; Methods), at a frame rate of $125 \mathrm{fps}$. The range of interest for SWCNT lengths goes from $0.5 \mu \mathrm{m}$ (the shortest optically resolvable length) to $2.5 \mu \mathrm{m}$ (the longest SWCNTs expected in the sample). For such lengths, the predicted fluorescence autocorrelation time under our experimental conditions goes from $30 \mathrm{~ms}$ to $2400 \mathrm{~ms}$, i.e., from 3.5 to 300 frames; the fast frame rate used was chosen in order to be able to observe the intensity fluctuations of the shortest optically resolvable SWCNTs.

Custom fitting and linking routines were used to localize long nanotubes (by fitting them to straight segments, the center of which were used as SWCNT positions) and reconstruct trajectories (figures 2b, 2c). Trajectories with less than 100 localizations were discarded, leaving $N=825$ trajectories.

Translational diffusion analysis was performed following standard single-particle tracking methodologies. When re- 


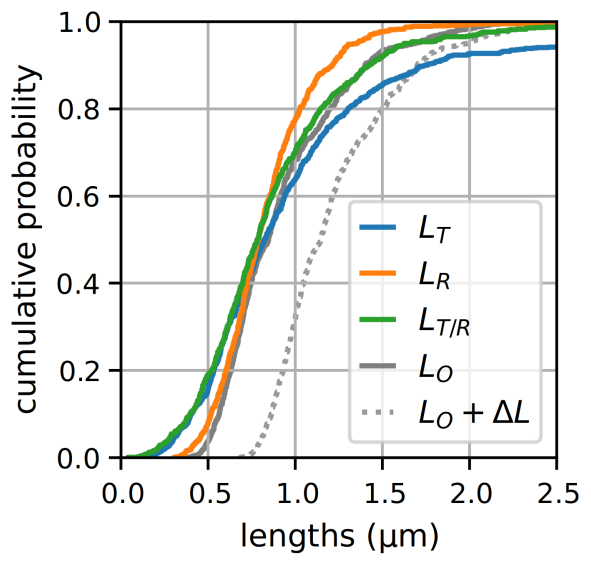

FIG. 3. Cumulative probability distributions of SWCNT lengths estimated from diffusion coefficients $\left(L_{T}\right.$ : translational diffusion; $L_{R}$ : rotational diffusion; $L_{T / R}$ : translational and rotational diffusion) and optically ( $L_{O}$ : without correction for exciton diffusion; $L_{O}+\Delta L$ : with correction for exciton diffusion).

quired, sample drift was estimated from the mean displacement of all nanotubes from each frame to the next and subtracted from all trajectories ${ }^{13}$.

The fluorescence autocorrelation function $\operatorname{ACF}(\Delta t)$ was computed for each trajectory and fitted, as for the simulations, to an exponential model. In the presence of uncorrelated experimental noise $\left(I_{\exp }(t)=I(t)+\varepsilon(t)\right)$, the experimental autocorrelation function is decreased by a constant multiplicative factor at all nonzero lags:

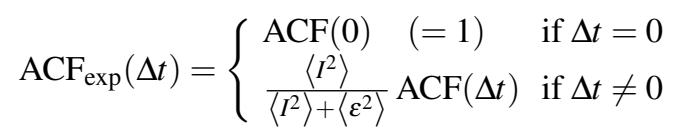

Therefore, experimental noise was taken into account by fitting this additional factor $\left(\left\langle I^{2}\right\rangle /\left(\left\langle I^{2}\right\rangle+\left\langle\varepsilon^{2}\right\rangle\right)\right)$.

For 18 of the 825 trajectories $(2 \%)$, the value of the autocorrelation function at the first lag was less than $\exp (-2)$. In these cases, we deemed the fluctuations to be too noisy or too fast to be resolved at the used frame rate. For the other 807 trajectories, for which the fluorescence autocorrelation time, and thus the rotational diffusion coefficient, could be estimated, we computed the three different diffusion-based length estimates: $L_{T}$, purely from the translational diffusion coefficient (i.e., the LAND estimate; eq. 4 ); $L_{R}$, purely from the rotational diffusion coefficient (eq. 7); and $L_{T / R}$, by combining translational and rotational diffusion coefficients (eq. 9). The $L_{T}$ and $L_{R}$ estimates depend on the solution viscosity and SWCNT hydronyamic diameter; here, we assumed that the solution viscosity matched its tabulated value $(\eta=20.6 \mathrm{mPas})$, and used a hydrodynamic diameter of $d=(5 \pm 2) \mathrm{nm}$. No thinfilm correction was applied to the solution viscosity, as imaging was performed at the center of a relatively thick, $\sim 60 \mu \mathrm{m}$ chamber. The $L_{T / R}$ estimate is fully independent of $\eta$, and only depends very weakly on $d$.

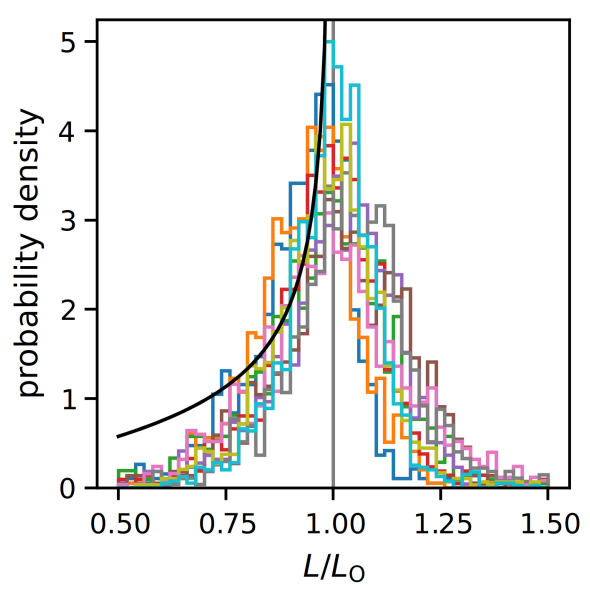

FIG. 4. Distribution of lengths $L$ measured on each frame of the trajectory, each normalized to its own half-sample mode $L_{O}$, for the 10 longest trajectories in the dataset, and (in black) theoretical distribution only considering geometrical effects and ignoring measurement inaccuracies described in the text. The asymmetry of the distributions is apparent.

The three diffusional methods yield overall distributions of SWCNT lengths with similar medians, but with different spreads (figure 3 ). Because $L_{T}$ and $L_{R}$ are computed from essentially independent data sources (SWCNT positions for $L_{T}$, SWCNT intensities for $L_{R}$ ), their consistency validates the accuracy of the measured distributions. Some differences, however, are present between the distributions: $L_{T}$ is the widest, $L_{R}$ is the sharpest, and $L_{T / R}$ is intermediate. The wider distribution of $L_{T}$ (i.e., of $D_{T}$ ) may arise from inaccurate localizations - occuring in particular when SWCNTs move partially out of focus in our thick sample, resulting in only one end of the SWCNT being taken into account by the localization fitter - or incomplete drift correction. Because such problems do not change the total collected fluorescence intensity, they do not affect $D_{R}$, which solely relies on SWCNT detection and trajectory reconstruction; therefore $L_{R}$ is a more accurate and sharper length estimate. $L_{T / R}$ is computed as a combination of $D_{T}$ and $D_{R}$, and is therefore affected to an intermediate degree by mislocalization and drift; any improvement to the estimation of $D_{T}$ would not only improve the precision of $L_{T}$ (assuming well-known $\eta$ and $d$ ), but also the precision of $L_{T / R}$ (independently of $\eta$ and $d$ ) as well.

Another length estimate which we considered for comparison with $L_{T}, L_{R}$ and $L_{T / R}$ is the direct measurement of the apparent SWCNT length on the movie images. Specifically, on a given frame, the apparent length $L$ of a SWCNT depends on its angle $\theta$ relative to the optical axis, $L=L_{O} \sin \theta$ (figure $1 \mathrm{a}$, where $L_{O}$ is the "optical" length of the nanotube when lying in the sample plane. The probability density of observing a certain length $L$ (only considering the SWCNT orientation randomness while ignoring measurement error) is thus

$$
p(L) \propto \sin \theta \frac{\mathrm{d} \theta}{\mathrm{d} L}=\frac{\tan \theta}{L_{O}},
$$


which diverges at $\theta=\pi / 2$, i.e. $L=L_{O}$. The experimentally observed distribution of lengths along a trajectory is affected by finite sampling, measurement errors, and worse detection efficiency for nanotubes aligned with the optical axis - which appear both shorter and dimmer - but is indeed asymmetric (figure 4) and is peaked at the true length $L_{O}$. Therefore, the nanotube length must be estimated as the mode of the distribution of observed SWCNT lengths along the trajectory. We used the half-sample mode method ${ }^{23}$ to obtain a nonparametric estimator of the mode of a finite sample. Finally, in order to take into account the SWCNT dark ends arising from exciton diffusion, a correction term $\Delta L \sim 2 \times 150 \mathrm{~nm}$ needs to be added to this optical estimate.

Strikingly, we find that the distribution of optical lengths including exciton diffusion correction $\left(L_{O}+\Delta L\right)$ is shifted by $\sim 300 \mathrm{~nm}$ relative to all diffusional length distributions (figure 3). (By coincidence, the uncorrected optical lengths $\left(L_{O}\right)$ matches the diffusional length distributions relatively well.) A possible reason for this discrepancy is the poor definition for the "ends" of the SWCNT in an optical image: at the SWCNT extremities, the fluorescence does not decrease sharply, but continously over one or two pixels (figure 2b), making sub-pixel determination of SWCNT length impossible in the absence of a detailed model for this decrease (the model also needs to take into account other sources of fluorescence decrease, such as partial defocus). Therefore, optical length measurements should be considered with caution, especially for quickly diffusing SWCNTs, when sub-pixel $(\lesssim 250 \mathrm{~nm})$ accuracy is required.

\section{CONCLUSION}

Here, we have described a simple method to improve the robustness of the SWCNT lengths obtained from a LAND analysis. The fluctuations in the fluorescence intensity of the SWCNT along its trajectory are directly correlated with the SWCNT rotational diffusion coefficient; by themselves, they yield a good estimate of the SWCNT length, which is fully independent of drift correction and other sources of localization error. As this estimate still depends on accurate knowledge of the solution viscosity and of the SWCNT hydrodynamic diameter, we propose yet another approach: we combine the fluorescence fluctuation analysis together with the translational diffusion analysis (which does depend on accurate localization) in order to obtain a length estimate that is fully independent of viscosity and nearly independent of the SWCNT hydrodynamic diameter. We foresee that this method will be a valuable improvement over the standard LAND approach for the high-throughput estimation of SWCNT lengths, required for many applications.

\section{METHODS}

\section{A. Sample preparation}

SWCNTs were prepared as previously described ${ }^{24}$ : HiPcosynthesized nanotubes (batch no. 195.7, Rice University) were mixed in $1 \% \mathrm{w} / \mathrm{v} \mathrm{NaDOC}$ in $\mathrm{D}_{2} \mathrm{O}$, homogenized in a shear mixer at $19000 \mathrm{rpm}$ for $10 \mathrm{~min}$, and tip sonicated at $20 \mathrm{~W}$ to $25 \mathrm{~W}$ power for $8 \mathrm{~min}$ in a cold water bath. SWCNT bundles and impurities were precipitated by ultracentrifugation at $144000 \times g$ for $4 \mathrm{hr}$.

A $1.5 \mu \mathrm{l}$ drop of SWCNT suspended in a $2: 1 \mathrm{v} / \mathrm{v}$ glycerolwater mixture was pressed between two \#1.5 coverslips, surrounded by vacuum grease, which formed a sealant preventing evaporation and convection. The thickness of solution between the coverslips was $\approx 60 \mu \mathrm{m}$, and imaging was performed close to the midpoint between the coverslips in order to minimize boundary effects ${ }^{16}$.

\section{B. Single SWCNT fluorescence video microscopy}

Imaging was performed on an inverted microscope (Nikon). $(6,5)$ SWCNTs were excited at the $S_{22}$ transition with a $568 \mathrm{~nm}$ laser (Coherent Sapphire). A quarter-wave plate was used to obtain a circularly polarized excitation beam. A longpass dichroic mirror (FF875-Di01, Semrock) was used on the excitation path, and a long-pass emission filter (ET900LP, Chroma) on the collection path. Images were collected through a water immersion $60 \times$ objective (NA 1.27, Nikon) on an InGaAs camera (C-RED2, First Light Imaging) at 125 frames per second.

\section{Analysis}

Data analysis (localization, trajectory reconstruction, LAND, fluorescence autocorrelation analysis) and simulations were performed using custom implementations of the described algorithms in the Python and $\mathrm{C}++$ programming languages.

\section{ACKNOWLEDGMENTS}

This work was performed with financial support from Agence Nationale de la Recherche (ANR-15-CE16-0004-03), ITMO Cancer AVIESAN within the framework of the Cancer Plan and the France-BioImaging National Infrastructure (ANR-10-INBS-04-01). A.L. acknowledges support from the Fondation ARC pour la recherche sur le cancer. 
${ }^{1}$ N. Behabtu, C. C. Young, D. E. Tsentalovich, O. Kleinerman, X. Wang, A. W. Ma, E. A. Bengio, R. F. ter Waarbeek, J. J. de Jong, R. E. Hoogerwerf, S. B. Fairchild, J. B. Ferguson, B. Maruyama, J. Kono, Y. Talmon, Y. Cohen, M. J. Otto, and M. Pasquali, "Strong, light, multifunctional fibers of carbon nanotubes with ultrahigh conductivity," Science 339, 182-186 (2013).

${ }^{2}$ A. D. Franklin, "Nanomaterials in transistors: From high-performance to thin-film applications," Science 349 (2015).

${ }^{3}$ X. He, H. Htoon, S. Doorn, W. Pernice, F. Pyatkov, R. Krupke, A. Jeantet, Y. Chassagneux, and C. Voisin, "Carbon nanotubes as emerging quantumlight sources," Nature Materials 17, 663-670 (2018).

${ }^{4}$ F. N. Soria, C. Paviolo, E. Doudnikoff, M.-L. Arotcarena, A. Lee, N. Danné, A. K. Mandal, P. Gosset, B. Dehay, L. Groc, L. Cognet, and E. Bezard, "Synucleinopathy alters nanoscale organization and diffusion in the brain extracellular space through hyaluronan remodeling," Nature Communications 11, 1-13 (2020).

${ }^{5}$ R. M. Williams, C. Lee, T. V. Galassi, J. D. Harvey, R. Leicher, M. Sirenko, M. A. Dorso, J. Shah, N. Olvera, F. Dao, D. A. Levine, and D. A. Heller, "Noninvasive ovarian cancer biomarker detection via an optical nanosensor implant," Science Advances 4, eaaq1090 (2018).

${ }^{6}$ S. Wang, Z. Liang, B. Wang, and C. Zhang, "Statistical characterization of single-wall carbon nanotube length distribution," Nanotechnology 17, 634 (2006).

${ }^{7}$ K. J. Ziegler, U. Rauwald, Z. Gu, F. Liang, W. Billups, R. H. Hauge, and R. E. Smalley, "Statistically accurate length measurements of single-walled carbon nanotubes," Journal of Nanoscience and Nanotechnology 7, 29172921 (2007).

${ }^{8}$ J. K. Streit, S. M. Bachilo, A. V. Naumov, C. Khripin, M. Zheng, and R. B. Weisman, "Measuring single-walled carbon nanotube length distributions from diffusional trajectories," ACS Nano 6, 8424-8431 (2012).

${ }^{9}$ N. Fakhri, F. C. MacKintosh, B. Lounis, L. Cognet, and M. Pasquali, "Brownian motion of stiff filaments in a crowded environment," Science 330, 1804-1807 (2010).

${ }^{10}$ A. Lee, K. Tsekouras, C. Calderon, C. Bustamante, and S. Pressé, "Unraveling the thousand word picture: an introduction to super-resolution data analysis," Chemical Reviews 117, 7276-7330 (2017).

${ }^{11}$ X. Michalet and A. J. Berglund, "Optimal diffusion coefficient estimation in single-particle tracking," Physical Review E 85, 061916 (2012).

${ }^{12}$ A. G. Godin, J. A. Varela, Z. Gao, N. Danné, J. P. Dupuis, B. Lounis, L. Groc, and L. Cognet, "Single-nanotube tracking reveals the nanoscale organization of the extracellular space in the live brain," Nature Nanotechnology 12, 238-243 (2017).

${ }^{13}$ C. Paviolo, F. N. Soria, J. S. Ferreira, A. Lee, L. Groc, E. Bezard, and L. Cognet, "Nanoscale exploration of the extracellular space in the live brain by combining single carbon nanotube tracking and super-resolution imaging analysis," Methods 174, 91-99 (2020).

${ }^{14}$ D. A. Tsyboulski, S. M. Bachilo, A. B. Kolomeisky, and R. B. Weisman, "Translational and rotational dynamics of individual single-walled carbon nanotubes in aqueous suspension," ACS Nano 2, 1770-1776 (2008).

${ }^{15}$ T. K. Cherukuri, D. A. Tsyboulski, and R. B. Weisman, "Length- and defect-dependent fluorescence efficiencies of individual single-walled carbon nanotubes," ACS Nano 6, 843-850 (2012).

${ }^{16} \mathrm{G}$. Li and J. X. Tang, "Diffusion of actin filaments within a thin layer between two walls," Physical Review E 69, 061921 (2004).

${ }^{17}$ V. R. N. Telis, J. Telis-Romero, H. Mazzotti, and A. L. Gabas, "Viscosity of aqueous carbohydrate solutions at different temperatures and concentrations," International Journal of Food Properties 10, 185-195 (2007).

${ }^{18}$ N.-S. Cheng, "Formula for the viscosity of a glycerol-water mixture," Industrial \& Engineering Chemistry Research 47, 3285-3288 (2008).

${ }^{19}$ A. Volk and C. J. Kähler, "Density model for aqueous glycerol solutions," Experiments in Fluids 59, 75 (2018).

${ }^{20}$ R. Duggal and M. Pasquali, "Dynamics of individual single-walled carbon nanotubes in water by real-time visualization," Physical Review Letters 96, 246104 (2006).

${ }^{21}$ N. Fakhri, D. A. Tsyboulski, L. Cognet, R. B. Weisman, and M. Pasquali, "Diameter-dependent bending dynamics of single-walled carbon nanotubes in liquids," Proceedings of the National Academy of Sciences 106, 1421914223 (2009).

${ }^{22}$ S. R. Aragon and D. Flamik, "High precision transport properties of cylinders by the boundary element method," Macromolecules 42, 6290-6299 (2009).

${ }^{23}$ D. R. Bickel and R. Frühwirth, "On a fast, robust estimator of the mode: Comparisons to other robust estimators with applications," Computational Statistics \& Data Analysis 50, 3500-3530 (2006).

${ }^{24}$ J. G. Duque, A. N. G. Parra-Vasquez, N. Behabtu, M. J. Green, A. L. Higginbotham, B. K. Price, A. D. Leonard, H. K. Schmidt, B. Lounis, J. M. Tour, S. K. Doorn, L. Cognet, and M. Pasquali, "Diameter-dependent solubility of single-walled carbon nanotubes," ACS Nano 4, 3063-3072 (2010). 